\title{
Functional Endoscopic Sinus Surgery in Patients with Chronic Rhinosinusitis and Polyposis and Asthma
}

\author{
${ }^{1}$ Nishi Sonkhya, ${ }^{2}$ Kalpana Sharma
}

\begin{abstract}
There has been an increasing awareness of the inter-relationship between inflammatory disease of the upper and lower airway. Both epidemiological and physiological data suggest that the respiratory tract, from the middle ear mucosa, through the nose and sinuses, and into the pulmonary tree behave as an integerated unit. Recent progress in understanding the biology of airway disease has identified that systemic inflammatory responses play a critical and integerating role in these diseases. The role of sinus surgery in the management of asthma patients has been a matter of debate for many years. We are presenting a well-designed prospective, controlled study in 50 patients with asthma and chronic rhinosinusitis (CRS) with nasal polyposis who were submitted to either functional endoscopic sinus surgery (FESS) or medical therapy.
\end{abstract}

Keywords: Chronic rhinosinusitis, Asthma, Functional endoscopic sinus surgery, ACT score.

How to cite this article: Sonkhya N, Sharma K. Functional Endoscopic Sinus Surgery in Patients with Chronic Rhinosinusitis and Polyposis and Asthma. Clin Rhinol An Int J 2014;7(2):52-57.

Source of support: Nil

Conflict of interest: None declared

\section{INTRODUCTION}

Over the past two decades, there has been an increasing awareness of the inter-relationship between inflammatory disease of the upper and lower airway. Both epidemiological and physiological data suggest that the respiratory tract, from the middle ear mucosa, through the nose and sinuses, and into the pulmonary tree behave as an integerated unit. ${ }^{1}$ Pathophysiological processes which affect one component of this integerated system often concurrently impact other portions of the airway, and overtime, isolated disease in one area can expand to involve other areas also. This interdependence of the upper and lower respiratory tracts has led to the concept of the unified airway, a model for

\footnotetext{
${ }^{1}$ Professor, ${ }^{2}$ Senior Resident

1,2Department of ENT, SMS Medical College and Hospitals Jaipur, Rajasthan, India

Corresponding Author: Nishi Sonkhya, Professor, Department of ENT, SMS Medical College and Hospitals, Jaipur, Rajasthan India, Phone: +91-1402518676, e-mail: drsonkhya@yahoo.co.in
}

understanding and framing inflammatory processes that affect the respiratory system. ${ }^{1}$

In an international multidisciplinary consensus meeting, the inter-relationship between the diseases of allergic rhinitis and asthma was examined thoroughly, and numerous epidemiological and pathophysiological links were found tying these two common conditions. In fact, the coexistence of these two processes was felt to be so frequent that this panel recommended that 'when considering a diagnosis of rhinitis or asthma, an evaluation of both the upper and lower airways should be made'. ${ }^{2}$

It is important to clarify and understand the relationship between diseases of the upper and lower respiratory tracts because of the prevalence of chronic rhinosinusitis, polyposis and asthma; their impact on patients' lives; the resultant burden on the health care system; and the potential benefits of an integrated management approach. Recent progress in understanding the biology of airway disease has identified that systemic inflammatory responses play a critical and integerating role in these diseases. Prospective, randomized, controlled studies are needed to better define the effects, that treatments for chronic rhinosinusitis have on asthma morbidity and health care costs.

The role of sinus surgery in the management of asthma patients has been a matter of debate for many years. Most published papers on this topic are retrospective and noncontrolled. We are presenting a well-designed prospective, controlled study in 50 patients with asthma and chronic rhinosinusitis (CRS) with nasal polyposis who were submitted to either functional endoscopic sinus surgery (FESS) or medical therapy.

\section{MATERIALS AND METHODS}

The present study was conducted in the ENT Department, SMS Medical College and Hospital, Jaipur from January 2011 to December 2011 (with 1 year follow-up). Patients presenting with CRS and polyposis and with asthma symptoms were evaluated in reference to nasosinus symptoms, endoscopic appearance of nasal cavity and osteomeatal complex (OMC), radiographic study (CT scan) of nose and PNS, asthma symptoms, pulmonary function by spirometry. 
Patients of age group (15-60 years) undergoing FESS for CRS with polyposis and also having asthma were included in the study group (25 cases). Patients of age group (15-60 years) who refused for surgery (FESS) for CRS and polyposis and having asthma (kept on medical treatment) were included in the control group ( 25 cases).

All patients underwent pretreatment and post-treatment (FESS $v s$ medical treatment) evaluation for asthma symptoms (ACT scores), night time awakenings, spirometry (FEV1), steroid doses, serum IgE, total eosinophil counts and nasal endoscopic score. Difference in proportions were compared by Chi-square test and difference in means were compared by the unpaired t-test.

Functional endoscopic sinus surgery was performed by the same surgeon following Messerklinger technique. Uncinectomy and middle meatal antrostomy was carried out in almost all patients. The exent of procedure was tailored to the extent of sinus disease as documented by CT scan and nasal endoscopic findings. Postoperative treatment protocol followed was broad spectrum antibiotics for 2 to 4 weeks with saline nasal douche twice a day for 8 weeks and fluticasone nasal spray 1 puff each nostril twice a day for 12 weeks. Post-treatment (FESS vs medical treatment) patients were followed up for 1 year. At 3, 6 and 12 months the same subjective and objective parameters were reassessed.

\section{RESULTS AND OBSERVATIONS}

There were $35(70 \%)$ males and $15(30 \%)$ females in our study. The age ranged from 15 to 60 years with $50 \%$ of patients falling into young age group i.e. 15 to 30 years. Maxillary sinus was involved in all cases. Seventeen out of 50 involved all the sinuses including maxillary, anterior ethmoids, posterior ethmoids, frontal and sphenoids. Unilateral involvement was seen in 6 cases. The extent of the surgery (FESS) was tailored according to the CT scan and nasal endoscopic findings in each individual (Table 1). Almost all patients presented with a history of nasal obstruction (49/50). Nasal discharge and post nasal drip were also common. Equal incidence was seen in almost all signs and symptoms in both the groups (Graph 1).

All the patients were assessed pretreatment (FESS/ medical treatment), at the end of 3, 6 and 12 months posttreatment to know the efficiency of the FESS/medical treatment on asthma control.

Effect of FESS/medical treatment on asthma symptoms (ACT score): The asthma control test (ACT) score is a simple and effective tool that uses some basic but important criteria to rate the level of control of asthma and track it over time. Patients were asked to fill a proforma containing questions regarding various symptoms of asthma, number of attacks, interference with activities, frequency of inhalers used and self rating of their asthma control over the last 4 weeks. Each answer was further divided into 5 grades and given the score from 1 to 5 in decreasing severity. So scores ranging from 5 to 25 were given to each patient according to severity. Significant improvement was observed in ACT scores in patients treated with FESS $(\mathrm{p}<0.001)$ (Table 2, Graph 2).

Effect of FESS/medical treatment on spirometry (FEV 1): It was observed that the FEV1 improved in study group while it was same or decreased in the control group. Significant improvement was observed in FEV1 $(\mathrm{p}<0.001)$ in study group (Table 3, Graph 3).

Effect of FESS /medical treatment on drug requirements for asthma control: In our study group, 23 patients were completely taken off from all sorts of anti-asthmatic drugs. In 2 patients, there was significant/substantial decrease in dosage of anti-asthmatic drugs. Only 10 patients had shown improvement in the control group (Graph 4).

Effect of FESS/medical treatment on nocturnal awakenings: It was observed that there was significant decrease in night time awakenings in study group after FESS $(\mathrm{p}<0.001)$. (Table 4, Graph 5) as compared to control group.

\section{DISCUSSION}

The companionship between asthma and sinusitis is confirmed in many studies, but the effect of sinusitis treatment on pulmonary status, especially the outcome of asthma after undergoing functional endoscopic sinus surgery, is still controversial. In most of the previous studies, selected subjective and objective variables were limited and could not entirely evaluate the outcome of sinus surgery in these patients. Also the studies were mostly not controlled. Therefore, the effects of functional endoscopic sinus surgery on subjective improvement of asthmatic patients with chronic sinusitis were evaluated.

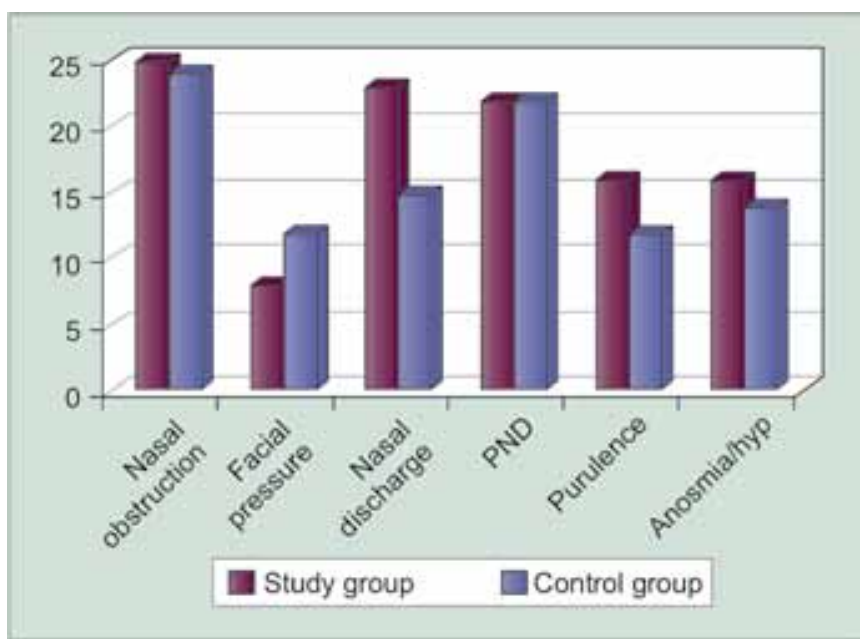

Graph 1: Presentation of symptoms in study and control group 


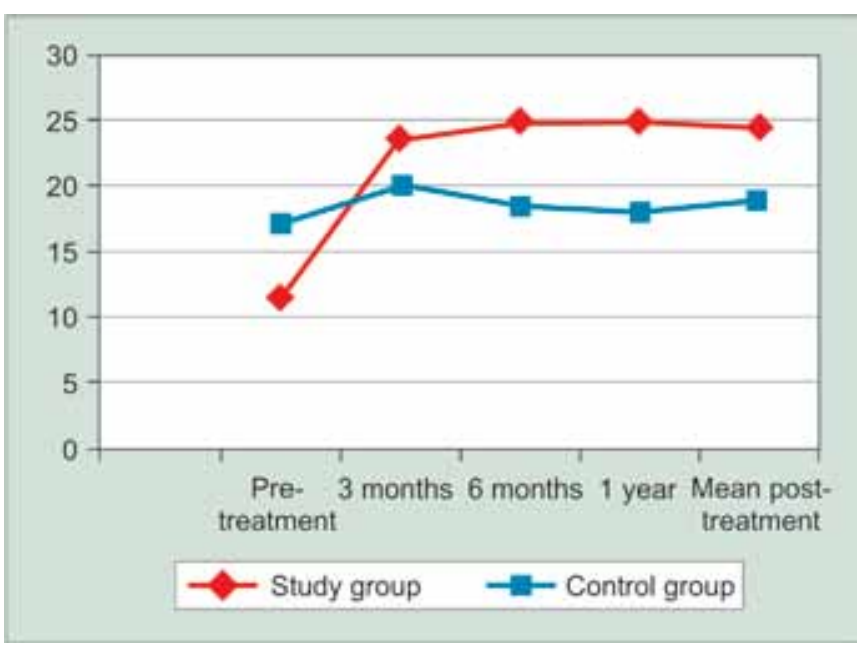

Graph 2: Pre and post-treatment ACT scores in study and control group

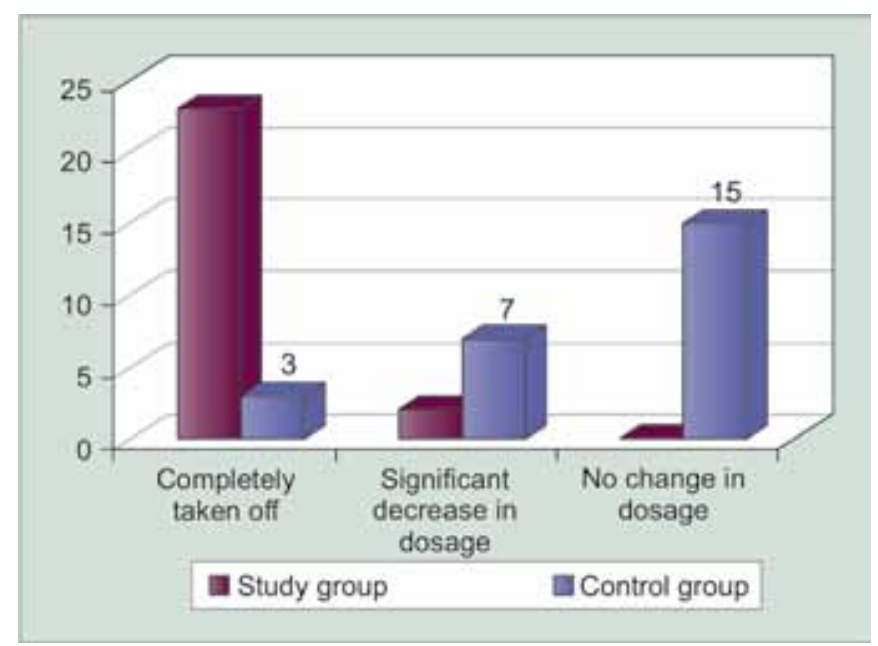

Graph 4: Post-treatment (FESS/medical treatment) changes in drug requirement for asthma control

Traditional and more radical, surgical approaches have shown good results in the treatment of chronic sinusitis with asthma. Because of improved understanding of pathogenesis of sinusitis, FESS has emerged as the surgical procedure of choice for chronic sinusitis. Functional endoscopic sinus surgery has been carried out in chronic sinusitis in asthma patients by a large number of workers. Stammberger ${ }^{3}$ in a study on 54 patients with asthma and sinusitis who underwent functional endoscopic sinus surgery reported slight to marked subjective as well as objective improvement in $83 \%$ of patients. Mean FEV1 showed significant improvement after FESS. Nishioka et $\mathrm{al}^{4}$ studied 20 asthma patients who underwent functional endoscopic sinus surgery for chronic sinusitis. Patients who had preoperative hospital admissions (4 patients) and emergency room or urgent physician office visits (18 patients) had a 75 and $81.3 \%$ reduction in visits, respectively, after surgery. Park et $\mathrm{al}^{5}$ in a study on seventynine patients with asthma and chronic sinusitis noted improvement in asthma in $80 \%$ of patients following FESS.

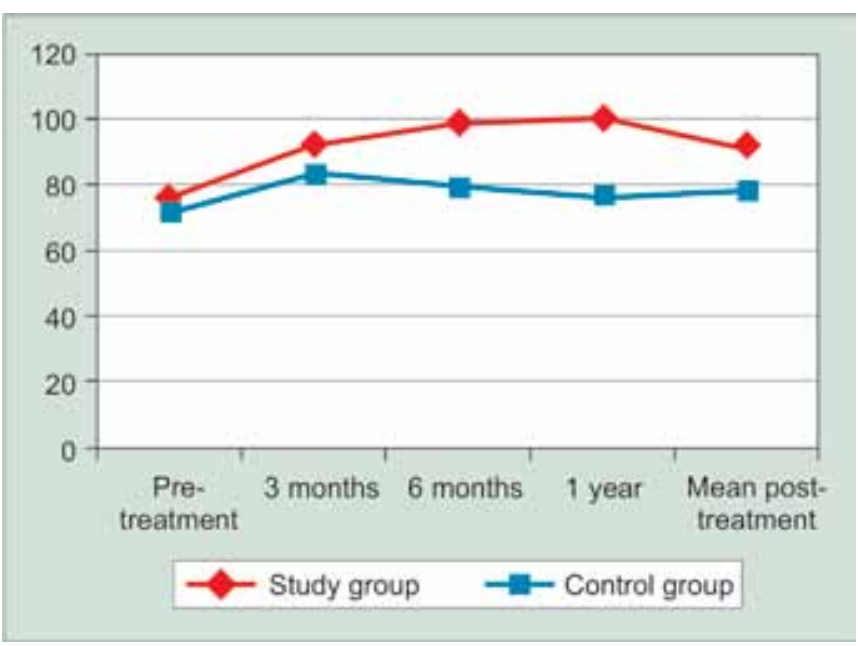

Graph 3: Pre and post-treatment results of spirometry (FEV-1)

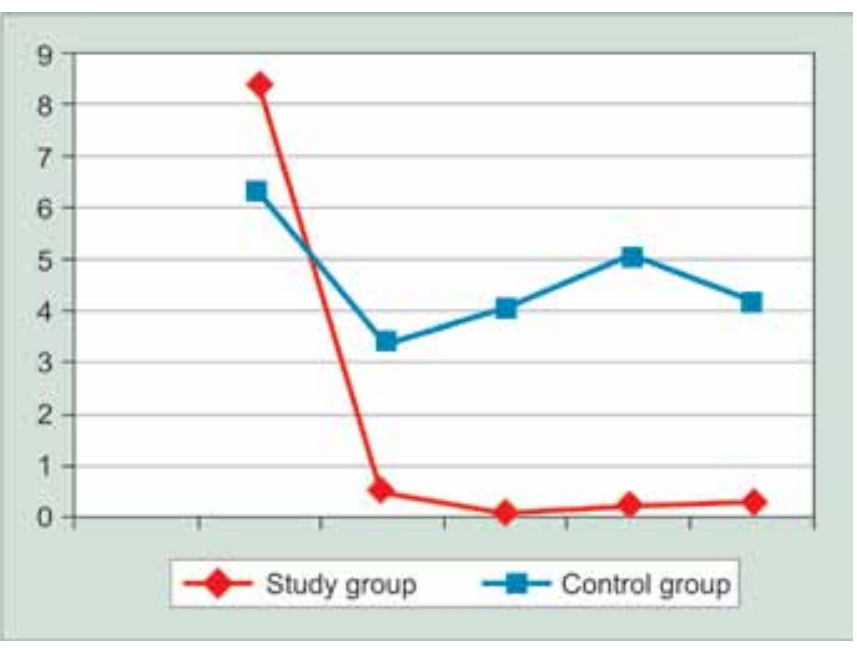

Graph 5: Significant reduction in night time awakenings in study group vs control group

Our aim of study was to determine whether or not FESS improves asthma symptoms in patients with moderately persistent asthma (uncontrolled), still symptomatic and are on inhalational steroids and to compare it with patient who refused for surgery and are kept on medical treatment. In conformity with the literature, in the present study significant improvement occurred in all the parameters noted.

Our results regarding the disease of the upper respiratory tract (CRS with NP), are in accordance with a long-term prospective randomized study by Row-Jones et $\mathrm{al}^{6}$ about the effectiveness of FESS on CRS with or without NP. In their study, there was a tendency for improvement of the overall asthma control in those who underwent FESS than those who were on medical treatment only. In our study, we observed improved asthma symptoms and overall good asthma control in the study group after FESS (Graph 2). A significant increase was recorded in ACT scores $(p<0.001)$ in patients in the study group (Table 2). 


\begin{tabular}{llll}
\hline & & Table 1: CT scan findings & \\
\hline CT scan findings & Study group & Control group & Grand total \\
\hline OMC, MS, AE & 5 & 5 & 10 \\
OMC, MS, AE, PE, FS, SS & 7 & 10 & 17 \\
OMC, MS, AE, FS & 4 & 2 & 6 \\
OMC, MS, AE, FS, SS & 6 & 4 & 10 \\
Rt (OMC, MS, AE, PE, FS, & 0 & 1 & 1 \\
SS) & & & 3 \\
Rt (OMC, MS, AE, FS, SS) & 0 & 3 & 1 \\
Rt (MS, AE, PE, FS, SS); & 1 & 0 & 2 \\
Lt (MS, AE) & 2 & & 50 \\
Rt (MS, AE, FS) & 25 & 0 & 25 \\
Grand total & & 25 & \\
\hline OMC: Osteomeatal
\end{tabular}

OMC: Osteomeatal complex block; MS: Maxillary sinusitis; AE: Anterior ethmoiditis; PE: Posterior ethmoiditis;

FS: Frontal sinusitis; SS: Sphenoidal sinusitis

Table 2: Asthma control text $(\mathrm{ACT})$ score $(\max .=25)$

\begin{tabular}{|c|c|c|c|c|c|}
\hline \multicolumn{2}{|l|}{ ACT scores } & Study group & Control group & $\begin{array}{l}p \text {-value } \\
\text { (unpaired t-test) }\end{array}$ & $L S$ \\
\hline \multicolumn{2}{|l|}{ Pretreatment } & $11.40 \pm 3.46$ & $17.04 \pm 1.79$ & $p<0.001$ & $5 \%$ \\
\hline \multirow[t]{3}{*}{ Post-treatment } & 3 months & $23.48 \pm 0.96$ & $20.12 \pm 1.78$ & $p<0.001$ & $5 \%$ \\
\hline & 6 months & $24.84 \pm 0.47$ & $18.40 \pm 2.29$ & $p<0.001$ & $5 \%$ \\
\hline & 1 year & $24.84 \pm 0.48$ & $17.92 \pm 2.21$ & $p<0.001$ & $5 \%$ \\
\hline \multicolumn{2}{|c|}{ Mean post-treatment } & $24.38 \pm 0.35$ & $18.81 \pm 2.33$ & $p<0.001$ & $5 \%$ \\
\hline
\end{tabular}

Table 3: Forced expiratory volume in $1 \mathrm{sec}$ (FEV-1)

\begin{tabular}{|c|c|c|c|c|c|}
\hline \multicolumn{2}{|l|}{ FEV 1\% } & \multirow{2}{*}{$\begin{array}{l}\text { Study group } \\
76.39 \pm 9.94\end{array}$} & \multirow{2}{*}{$\begin{array}{l}\text { Control group } \\
71.85 \pm 9.63\end{array}$} & \multirow{2}{*}{$\begin{array}{l}p \text {-value } \\
\text { (unpaired t-test) } \\
p<0.05\end{array}$} & \multirow{2}{*}{$\begin{array}{l}L S \\
5 \%\end{array}$} \\
\hline Pretreatment & & & & & \\
\hline \multirow[t]{3}{*}{ Post-treatment } & 3 months & $92.3 \pm 4.94$ & $83.98 \pm 8.60$ & $p<0.001$ & $5 \%$ \\
\hline & 6 months & $98.22 \pm 3.15$ & $79.97 \pm 7.51$ & $p<0.001$ & $5 \%$ \\
\hline & 1 year & $100.37 \pm 2.78$ & $76.93 \pm 9.48$ & $p<0.001$ & $5 \%$ \\
\hline \multicolumn{2}{|c|}{ Mean post-treatment } & $91.99 \pm 10.98$ & $78.18 \pm 5.11$ & $p<0.001$ & $5 \%$ \\
\hline
\end{tabular}

Table 4: Night time awakenings

\begin{tabular}{|c|c|c|c|c|c|}
\hline \multicolumn{2}{|c|}{ Night time awakenings/week } & \multirow{2}{*}{$\begin{array}{l}\text { Study group } \\
8.3 \pm 1.8\end{array}$} & \multirow{2}{*}{$\begin{array}{l}\text { Control group } \\
6.32 \pm 1.93\end{array}$} & \multirow{2}{*}{$\begin{array}{l}p \text {-value } \\
\text { (unpaired t-test) } \\
\mathrm{p}<0.001\end{array}$} & \multirow{2}{*}{$\begin{array}{l}L S \\
5 \%\end{array}$} \\
\hline Pretreatment & & & & & \\
\hline \multirow[t]{3}{*}{ Post-treatment } & 3 months & $0.48 \pm 0.5$ & $3.4 \pm 1.58$ & $p<0.001$ & $5 \%$ \\
\hline & 6 months & $0.08 \pm 0.28$ & $4.04 \pm 1.67$ & $p<0.001$ & $5 \%$ \\
\hline & 1 year & $0.24 \pm 0.52$ & $5.08 \pm 1.80$ & $p<0.001$ & $5 \%$ \\
\hline \multicolumn{2}{|c|}{ Mean post-treatment } & $0.27 \pm 0.52$ & $4.17 \pm 1.71$ & $p<0.001$ & $5 \%$ \\
\hline
\end{tabular}

In another study, 23 of 24 patients with simultaneous chronic sinusitis and asthma experienced a $75 \%$ or greater improvement in asthma symptoms after surgical drainage. ${ }^{7}$ Mings and colleagues, ${ }^{8}$ showed that $62 \%$ of 16 patients who underwent bilateral sphenoethmoidectomy after not responding to aggressive medical treatment of sinusitis had subjective improvement of their asthma. Eighty percent of patients reported a significantly reduced prednisolone requirement. One report presented results for 205 steroid dependent patients with aspirin triad. After FESS, 40\% of the patients were able to discontinue steroid; another $44 \%$ had their illness controlled with alternate day steroid therapy or bursts. ${ }^{9}$ Manning et al. ${ }^{10}$ studied 14 children with severe asthma requiring at least intermittent systemic steroid therapy. Eleven of 14 patients who underwent FESS demonstrated a significant reduction in hospitalization and school days missed. In our study 23 of 25 (92\%) patients were able to put off their steroids for asthma while in the control group only 3 (12\%) patients were able to discontinue their medications and $15(60 \%)$ experienced no change in the dosage of steroids (Graph 4).

Dunlop et $\mathrm{a}^{11}$ in a study of 50 asthmatic patients with a history of chronic rhinosinusitis with or without NP reported statistically significant improvement in the postoperative need for hospitalization $(p=0.225)$, and the postoperative need for oral steroids $(p=0.202)$, but not in the overall asthma control. We noted a significant improvement in our study group in terms of postoperative need for steroid 
inhalers $(\mathrm{p}<0.001)(\mathrm{Graph} 4)$ and overall asthma control (ACT scores, $\mathrm{p}<0.001$ ).

Uri et $\mathrm{al}^{12}$ reported that in a group of 34 asthmatic patients with massive polyposis, FESS did not improve the asthma state, but had a statistically significant effect in prednisolone intake $(p=0.046)$, and inhalations of bronchodilators $(\mathrm{p}=0.001)$. Batra et $\mathrm{al}^{13}$ reported in a group of 17 patients with CRS, nasal polyposis and concomitant, oral steroids dependent, asthma; statistically significant improvement occured in pulmonary function test FEV1 $(\mathrm{p}<0.014)$ and use of oral steroids ( $p=0.048$ ). In our study, we also observed significant improvement in overall asthma control in terms of ACT scores (Table 2, Graph 2), spirometry (Table 3, Graph 3) steroid doses (Graph 4), and night time awakenings (Table 4, Graph 5). In our study better asthma control is attributed to the meticulous surgery performed in order to eradicate the disease burden from the sinuses, as well as educating the patients for avoidance of the allergen.

In a unique study that included treatment randomization, Ragab et $\mathrm{al}^{14}$ looked at the subgroup of CRS patients with asthma who were part of a larger study in which the patients were randomized to receive either medical or surgical treatment for their CRS. Their results confirmed that successful treatment of CRS was associated with improved control of asthma. Gulati et $a 1^{15}$ reported in a group of 25 patients with CRS and asthma who underwent FESS, a significant reduction in the number of hospitalizations for asthma, night time awakenings, as well as a decrease in the mean use of bronchodilator inhalers. The mean FEV1 observed postoperatively showed significant increase $(p<0.001)$. Although, their study was uncontrolled for the effect of intervention, i.e. FESS used. We observed in our prospective, controlled study that the study group undergoing FESS showed a significant decrease in the use of anti-asthmatic drugs, overall asthma control including ACT scores $(\mathrm{p}<0.001)$ and FEV1 $(p<0.001)$. While the patients on medical treatment showed improvement initially but not in the longrun.

Senior et $\mathrm{a}^{16}$ concluded in their study that FESS in 30 patients with CRS and asthma had positive impact in $89.9 \%$, no change was reported in $11.1 \%$, while no patients reported worsening of the subjective measurements for asthma. Regarding objective measurements, $46 \%$ reported improvement in bronchodilator inhalers use and $65 \%$ reported improvement in oral steroids use. Park et $\mathrm{al}^{17}$ reported in their study in 79 patients with CRS and asthma, 80\% improvement in the subjective measurements for asthma and significant improvement in the need for hospitalization and use for oral steroids for asthma. We noted improvement in almost all patients in our study group objectively as well as subjectively. There was a considerable decrease in use of anti-asthmatic drugs with FESS (Graph 4).
The influence of FESS in patients with asthma is attributed to several mechanisms that seem to explain the link between upper and lower airways. This surgical approach aims at the restoration of normal nasal function (filtration, humidification, heating of incoming air), thus protecting from irritation of the lower airways. It is also well known that upper and lower airways communicate with several mechanisms, such as neural reflexes and systemic pathways. Functional endoscopic, sinus surgery positively influences the amount of inflammatory mediators that can induce symptoms in the unified airways, reduce beta adrenergic blockade related to chronic rhinosinusitis and reduce postnasal drip. On the other hand, certain authors reported that sinus surgery has a negative effect upon asthma. Lamblin et $a l^{18}$ in their study concluded that sinus surgery had negative impact upon objective measurements (Spirometry) of bronchial function. While in our study FESS improved all the objective as well as subjective parameters. There is definitely a positive impact of FESS on asthma upon objective measurements of asthma FEV1 $(p<0.001)$.

Our clinical study supports the hypothesis that FESS has a positive influence in the lower airways function of the asthmatic patients preventing from triggering asthma symptoms. Recorded improvement of the subjective and objective parameters measured, should not only be attributed to alleviation of upper airway symptoms and to concomitant improvement of a patient's quality of life, but to the positive influence of FESS on the intrinsic mechanisms, which probably control the link between upper and lower respiratory tract.

Nevertheless, our study was controlled, adding to it is better interpretation of the results as compared to the previous studies, a larger sample size and longer follow-up is required to completely define the diverisity of variables.

In spite of our results regarding the effect of sinus surgery on improving sinusitis and asthma, a longer follow-up period may illustrate different results. Therefore, studies with longer follow-up periods should be done in future. Also, comparing the outcome of asthma in different sinusitis pathologies requires larger sample sizes to completely define the diversity of the variables.

\section{REFERENCES}

1. Passalacqua G, Ciprandi G, Canonica GW. United Airway diseases: therapeutic aspects. Thorax 2000;55:S26-S27.

2. Bousquet J, van Cauwenberge $P$, Khaltaev N, et al. Allergic rhinitis and its impact on asthma (ARIA): executive summary of the workshop report. Allergy 2002;57:841-855.

3. Stammberger H, Kopp W, Decornfeld J, Hawkw M. Functional endoscopic sinus surgery. The Messerklinger Technique. Philadelphia 1991; B,O, Decker 453-457.

4. Nishioka GJ, Cook PR, Davis WE, Mckansey JP. Functional endoscopic sinus surgery in patients with chronic sinusitis and asthma. Otolaryngol Head Neck Surg 1994;110:494-500. 
5. Park AH, Lau J, Stankiewicz J, Chow J. The role of functional endoscopic sinus surgery in asthmatic patients. J Otolaryngol 1998;27:275-280.

6. Rowe-Jones JM, Medcalf M, Durham SR, et al. Functional endoscopic sinus surgery: 5 year follow-up and results of a prospective, randomised, stratified, double-blind, placebo controlled study of postoperative fluticasone propionate aqueous nasal spray. Rhinology 2005;43:2-10.

7. Davidson F. Chronic sinusitis and infectious asthma. Arch Otolaryngol 1969;90:292-307.

8. Mings R, Friedman WH, Linford P, et al. Five year follow-up of the effects of bilateral intranasal sphenoethmoidectomy in patients with sinusitis and asthma. Am J Rhinol 1988;71:123-132.

9. English GM. Nasal polypectomy and sinus surgery in patients with asthma and aspirin idiosyncrasy. Laryngoscope 1986;96:371-380.

10. Manning SC, Wasserman RL, SilverR, Phillips DL. Results ofendoscopic sinus surgery in pediatric patients with chronic sinusitis and asthma. Arch Otolaryngol Head Neck Surg 1994; 120:1142-1145.

11. Dunlop G, Scadding GK, Lund VJ. The effect of endoscopic sinus surgery on asthma: management of patients with chronic rhinosinusitis, nasal polyposis and asthma. Am J Rhinol 1999;13:261-265.
12. Uri N, Cohen-Kerem R, Barzilai G, et al. Functional endoscopic sinus surgery in the treatment of massive polyposis in asthmatic patients. J Laryngol Otol 2002;116:185-189.

13. Batra PS, Kern RC, Tripathi A, et al. Outcome analysis of endoscopic sinus surgery in patients with nasal polyps and asthma. Laryngoscope 2003;113:1703-1706.

14. Ragab S, Scadding GK, Lund VJ, et al. The treatment of chronicrhinosinusitis and its effect on asthma. Eur Resp J 2006;28: 68-74.

15. Gulati SP, et al. The role of FESS in patients with asthma with chronic sinusitis. Indian J Otolaryngol Head Neck Surg 2008 April-June;60:152-155.

16. Senior BA, Kennedy DW, Tanabodee JC, et al. Long-term impact of functional endoscopic sinus surgery on asthma. Otolaryngol Head Neck Surg 1999;121:66-68.

17. Park AH, Lau J, Stankiewicz J, et al. The role of functional endoscopic sinus surgery in asthmatic patients. J Otolaryngol 1998;27:275-280.

18. Lamblin C, Brichet A, Perez T, et al. Long-term follow-up of pulmonary function in patients with nasal polyposis. Am J Respiratory Crit Care Med 2000;161:406-413. 